\title{
Má absorção de lactose em crianças e adolescentes: diagnóstico através do teste do hidrogênio expirado com o leite de vaca como substrato
}

\author{
Lactose malabsorption in children and adolescents: \\ diagnosis through breath hydrogen test using cow milk
}

\author{
Fernanda M. Pretto ${ }^{1}$, Themis R. Silveira ${ }^{2}$, Virginia Menegaz ${ }^{3}$, Jarbas de Oliveira ${ }^{4}$
}

\section{Resumo}

Objetivo: determinar a prevalência de má absorção de lactose e sua associação com a cor da pele e com a idade em crianças e adolescentes de escolas públicas do município de Porto Alegre.

Métodos: foi realizado um estudo transversal, que incluiu 225 indivíduos de 8 a 18 anos, alunos de duas escolas públicas do município de Porto Alegre. A seleção dos alunos ocorreu através de sorteio. Os participantes foram classificados segundo a cor da pele (brancos e não-brancos), e a faixa etária (8 a 12 e 13 a 18 anos). A má absorção de lactose foi diagnosticada através do teste do hidrogênio expirado após ingestão de $250 \mathrm{ml}$ de leite de vaca integral industrializado. $\mathrm{O}$ teste teve duração de 3 horas, com coletas em jejum e aos 60, 120 e 180 minutos após a ingestão do leite. Foi considerado como critério de positividade o aumento $\geq 20 \mathrm{ppm}$ na concentração de hidrogênio em relação ao nível basal.

Resultados: foram estudados 225 alunos, com uma média e desvio-padrão de idade de $12,2 \pm 2,0$ anos. Cento e trinta e quatro indivíduos eram do sexo feminino $(59,6 \%)$. Cento e cinqüenta e quatro alunos eram de cor branca $(68,4 \%)$ e os restantes, de cor nãobranca. A má absorção de lactose foi evidenciada em 19/225 casos $(8,4 \%)$. Ela foi diagnosticada em $8 / 154$ alunos de cor branca $(5,2 \%)$ e em 11/71 alunos de cor não-branca $(15,5 \%)(p=0,02)$. Em relação à faixa etária, ocorreram $15 / 143$ casos de má absorção nos alunos entre 8 a 12 anos (10,5\%), e 4/82 casos entre 13 e 18 anos (4,9\%) (p= $0,227)$.

Conclusões: a prevalência de má absorção de lactose encontrada em alunos de escolas públicas de Porto Alegre é significativa, especialmente se considerarmos que foram utilizadas doses fisiológicas do dissacarídeo ( $250 \mathrm{ml}$ de leite) para o diagnóstico. A taxa de má absorção foi maior entre as crianças de cor não-branca em relação às crianças de cor branca, confirmando a influência racial na hipolactasia primária do tipo adulto. Não foi encontrada associação entre má absorção de lactose e faixa etária.

J Pediatr (Rio J) 2002; 78 (3): 213-18: má absorção de lactose, leite de vaca, teste do hidrogênio expirado.

\begin{abstract}
Objective: to determine the prevalence of lactose malabsorption and its association with skin color and age in children and teenagers attending public schools in Porto Alegre, Brazil.

Methods: a cross-sectional study was performed with 225 subjects between 8 and 18 years attending two public schools in Porto Alegre, Brazil. Patients were randomly selected. Subjects were classified according to skin color (white and nonwhite) and age group ( 8 to 12 and 13 to 18 years). Lactose malabsorption was diagnosed using the breath hydrogen test after ingestion of $250 \mathrm{ml}$ of whole cow milk. The test lasted for 3 hours, with collections after fasting and 60 , 120, and 180 minutes after milk ingestion. Malabsorption was determined in the presence of increase of $\geq 20 \mathrm{ppm}$ in hydrogen concentration regarding the basal levels.

Results: two-hundred and twenty-five students were studied, with a mean age \pm standard deviation of $12.2 \pm 2.0$ years. The subjects consisted of 134 females (59.6\%); 154 white (68.4\%); and 71 nonwhite. Lactose malabsorption was observed in $19 / 225$ cases (8.4\%). It was diagnosed in $8 / 154$ white patients $(5.2 \%)$ and in $11 / 71$ nonwhite patients $(15.5 \%)(\mathrm{P}=0.02)$. Regarding the age group, we found $15 / 143$ cases of malabsorption in students between 8 and 12 years $(10.5 \%)$, and $4 / 82$ cases in students between 13 and 18 years (4.9\%) $(\mathrm{P}=0.227)$.

Conclusions: the prevalence of lactose malabsorption in students attending public schools in Porto Alegre is significant, especially if we consider that the physiological doses $(250 \mathrm{ml}$ of milk) were used for diagnosis. The malabsorption rate was higher among nonwhite children, which confirms the influence of race on primary adult type hypolactasia. No association was observed between lactose malabsorption and age group.
\end{abstract}

J Pediatr (Rio J) 2002; 78 (3): 213-18: lactose malabsorption, cow milk, breath hydrogen test.

1. Mestre em Pediatria pela UFRGS

2. Professora Adjunta do Dep. de Pediatria da Faculdade de Medicina da UFRGS. Mestre e Doutora. Coordenadora do Programa de Transplante Hepático Infantil do Hospital de Clínicas de Porto Alegre (HCPA).

3. Acadêmica de Nutrição da UFRGS.

4. Professor Titular da Faculdade de Biociências da PUC-RS. Doutor.

Fonte Financiadora: Fundo de Incentivo à Pesquisa do HCPA.

Artigo submetido em 21.11.01, aceito em 13.03.02. 


\section{Introdução}

Durante o processo de digestão, a lactose deve ser hidrolisada no intestino delgado nos dois monossacarídeos que a compõem: glicose e galactose. Esses produtos serão absorvidos através de transporte ativo dependente de sódio e mediado por carreador ${ }^{1}$. A hidrólise da lactose é realizada por uma betagalactosidase, conhecida como lactase.

Na maioria da população mundial, após o desmame, há um declínio gradual na atividade da lactase ${ }^{1}$. Esse fenômeno, denominado hipolactasia primária do tipo adulto, é a forma mais comum de deficiência de dissacaridase determinada geneticamente ${ }^{1}$. A persistência da atividade enzimática na vida adulta tem herança autossômica dominante, enquanto a hipolactasia é herdada de forma autossômica recessiva ${ }^{2,3}$. A má absorção de lactose é o fenótipo predominante nas populações nativas da Austrália, Oceania, leste e sudeste da Ásia, África Tropical e Américas ${ }^{1}$.

O declínio nos níveis de lactase é progressivo durante a infância e a adolescência, havendo um aumento nas taxas de má absorção de acordo com a idade ${ }^{4-6}$. Estudos de prevalência de má absorção de lactose demonstram taxas menores de má absorção em indivíduos de cor branca em relação aos indivíduos de cor preta, parda ou amarela ${ }^{7,8}$. As doses e formas de administração da lactose, utilizadas para o diagnóstico, também influenciam as taxas de prevalência. Leis et al. ${ }^{6}$, em estudo que incluiu 850 indivíduos de 3 a 85 anos, demonstrou 32,5\% de má absorção com a administração de $2 \mathrm{~g} / \mathrm{kg}$ de lactose em solução aquosa a $20 \%$. Os indivíduos com testes positivos após essa dose repetiram o exame com a utilização de $250 \mathrm{ml}$ de leite e $250 \mathrm{ml}$ de iogurte. As taxas de má absorção encontradas foram de 13,7 e $3,8 \%$, respectivamente.

Atualmente, o teste do hidrogênio expirado é uma das técnicas mais empregadas no diagnóstico da má absorção de lactose ${ }^{9}$. A fermentação da lactose não absorvida pela flora colônica resulta na produção de hidrogênio. Parte desse gás será eliminado pelos pulmões, podendo ser detectado no ar expirado. $\mathrm{O}$ aumento na concentração de hidrogênio, em amostras de ar expirado, após a administração de lactose, é indicativo de má absorção e fermentação desse carboidrato, uma vez que não existem outras fontes endógenas para a produção de hidrogênio nos mamíferos ${ }^{10}$. A dose-padrão utilizada nesse teste é de $2 \mathrm{~g} / \mathrm{kg}$ de lactose em solução aquosa a $20 \%$. Contudo, como o teste do hidrogênio expirado pode detectar até 2 gramas de lactose não absorvida, alguns autores têm proposto o uso de doses menores e mais fisiológicas de lactose, assim como o uso do leite ou de iogurte como veículos ${ }^{6,11}$.

No Rio Grande do Sul, não existem estudos publicados sobre má absorção de lactose em crianças. A extrapolação de dados obtidos em outras regiões brasileiras é prejudicada pelas diferenças na miscigenação racial, que influenciam as taxas de prevalência.

Este estudo tem como objetivo determinar, através do teste do hidrogênio expirado, a prevalência de má absorção de doses fisiológicas de lactose e sua associação com a cor da pele e a faixa etária em crianças e adolescentes de escolas públicas, do município de Porto Alegre.

\section{Métodos}

Um estudo transversal foi conduzido no período compreendido entre maio e agosto de 2000. A população em estudo foi constituída por crianças e adolescentes de 8 a 18 anos de idade, de escolas públicas do município de Porto Alegre.

Duas escolas, situadas na zona norte de Porto Alegre, foram escolhidas para participar da pesquisa. Essas escolas apresentavam um grande número de turmas no turno da manhã, sendo incluídos apenas os alunos desse turno, uma vez que os exames eram realizados durante as atividades escolares, e os indivíduos selecionados deveriam comparecer com $8 \mathrm{~h}$ de jejum, o que dificultaria coletas no turno da tarde. Além disso, escolhemos escolas situadas na periferia de Porto Alegre, nas quais encontraríamos uma maior proporção de indivíduos de cor não-branca. A seleção dos alunos ocorreu através de sorteio pelo número de cada aluno na lista da turma. Os alunos sorteados recebiam o termo de consentimento, que deveria ser trazido assinado pelos pais ou responsáveis por ocasião da realização do exame. Foram excluídos os indivíduos com as seguintes condições: a) diagnóstico prévio de doença com contraindicação ao uso de leite; b) tempo de jejum inferior a 8 horas; c) uso de antibióticos, enemas ou laxativos nos quinze dias precedentes ao exame; d) diarréia no dia da realização do exame ou nos sete dias precedentes; e) fumo nas seis horas precedentes ao exame ou durante a realização do mesmo; f) adormecimento durante o exame; g) inabilidade para a realização adequada do exame; h) não concordância em participar do estudo por parte do aluno ou a não assinatura do termo de consentimento por um dos pais ou responsáveis.

De cada participante da pesquisa, era preenchida uma ficha com nome completo, idade, cor da pele e dados antropométricos. Os alunos foram classificados de acordo com a cor da pele em brancos e não-brancos. Foram também divididos em duas faixas etárias: de 8 a 12 anos e de 13 a 18 anos. Calculamos dois indicadores do estado nutricional para cada aluno: peso/idade e altura/idade, e a comparação da amostra com o padrão de referência (no caso, as curvas produzidas pelo National Center of Health Statistics) foi realizada através do escore $\mathrm{z}^{12}$. Foram classificados como desnutridos os alunos com escore $\mathrm{z}$ igual ou inferior $\mathrm{a}-2$.

Partindo-se de uma prevalência esperada de má absorção de lactose de $10 \%$ entre as crianças e/ou adolescentes de cor branca e de $30 \%$ entre os de cor não-branca $(R P \geq 3)$, o tamanho amostral calculado foi de aproximadamente 150 indivíduos para a: 0,05 e b: 0,20. Com este número, também poderíamos estimar a prevalência de má absorção de lactose com uma margem de erro de $6 \%$. 
O teste do hidrogênio expirado era realizado em duas etapas: coleta e armazenamento do material biológico nas escolas, e análise dos níveis de hidrogênio através de cromatografia gasosa no Hospital de Clínicas de Porto Alegre. O aparelho de cromatografia gasosa utilizado para medida da concentração de hidrogênio no ar expirado foi o Quintron Microlyzer modelo 12i.

Para coleta e armazenamento das amostras de ar expirado, foram utilizados um sistema coletor e recipientes fornecidos pela Quintron ${ }^{\circledR}$. A primeira coleta era realizada no início da manhã, após jejum de 8 horas. A seguir, cada participante recebia um copo de $250 \mathrm{ml}$ de leite de vaca integral industrializado, correspondente a $12,5 \mathrm{~g}$ de lactose. As coletas subseqüentes eram realizadas aos 60,120 e 180 minutos após a ingestão do leite. A má absorção de lactose foi diagnosticada quando o aumento máximo da concentração de hidrogênio (delta $\mathrm{H}_{2}$ ) foi igual ou superior a 20 partes por milhão (ppm). $\mathrm{O}$ delta $\mathrm{H}_{2}$ foi calculado como a diferença entre o valor basal e o maior valor da concentração de hidrogênio obtido durante o exame.

Os dados foram processados e analisados por meio dos programas Epi-Info versão 6.04, PEPI versão 3.0 e SPSS versão 10.0. As variáveis quantitativas foram descritas através de medidas de tendência central (média e mediana) e dispersão (desvio-padrão), e as variáveis categóricas foram expressas por percentuais. A comparação das variáveis categóricas foi realizada mediante o teste do Quiquadrado com correção de Yates. No caso da comparação das médias das variáveis contínuas, foi adotado o teste $t$ de Student. Considerou-se como diferença significativa um valor de $p$ inferior a 0,05 .

O protocolo do presente estudo e seu termo de consentimento informado foram aprovados pela Comissão Científica e de Ética do Hospital de Clínicas de Porto Alegre.

\section{Resultados}

Trezentos e oito alunos foram sorteados e receberam o termo de consentimento para participar do estudo, dos quais, 83 foram excluídos, sendo 66 por não terem apresentado o termo de consentimento assinado pelos responsáveis no dia da realização do exame, 5 por não terem comparecido em jejum, 5 por uso recente de antibióticos, 4 por terem apresentado diarréia e 3 por terem se alimentado durante as coletas. Na faixa etária dos 8 aos 12 anos, 178 foram sorteados e 32 excluídos (18\%), enquanto, dos 13 aos 18 anos, 130 foram sorteados e 51 excluídos ( $39 \%$ ) $(\mathrm{p}<0,001)$.

Dos 225 indivíduos efetivamente incluídos no estudo, $134(59,6 \%)$ eram do sexo feminino. A idade variou de 8 a 18 anos, com média de $12,1 \pm 2,0$ anos e mediana de 11,8 anos. Cento e cinqüienta e quatro alunos $(68,4 \%)$ eram de cor branca, e 71 (31,6\%) de cor não-branca (no caso, de cor preta ou parda). Em relação ao estado nutricional, a média do escore $\mathrm{z}$ foi de $0,16 \pm 1,42$ para peso/idade e de $-0,17 \pm$ 0,97 para altura/idade. Cinco alunos $(2,2 \%)$ foram conside- rados desnutridos (escore $\mathrm{z}$ igual ou inferior a -2 para peso/idade e/ou altura/idade). Os dados antropométricos só não foram obtidos de um dos alunos.

A comparação entre os alunos de cor branca e nãobranca em relação à média de idade, sexo e estado nutricional não apresentou diferenças significativas (Tabela 1).

Tabela 1 - Características gerais dos alunos de acordo com a cor

\begin{tabular}{|c|c|c|c|}
\hline Variáveis & $\begin{array}{c}\text { Brancos } \\
\text { n }(\%)\end{array}$ & $\begin{array}{c}\text { Não-brancos } \\
\text { n (\%) }\end{array}$ & $\mathbf{p}$ \\
\hline \multicolumn{4}{|l|}{ Sexo } \\
\hline Masculino & $61(39,6)$ & $30(42,2)$ & 0,819 \\
\hline Feminino & $93(60,4)$ & $41(57,8)$ & \\
\hline \multicolumn{4}{|l|}{ Idade (anos) } \\
\hline Média \pm desvio-padrão & $12,1 \pm 1,9$ & $12,2 \pm 2,0$ & 0,559 \\
\hline \multicolumn{4}{|c|}{ Índice (escore $z \pm$ desvio-padrão) } \\
\hline Peso/idade & $-0,01 \pm 1,08$ & $0,25 \pm 1,08$ & 0,108 \\
\hline Altura/idade & $-0,23 \pm 0,96$ & $-0,03 \pm 1,00$ & 0,160 \\
\hline
\end{tabular}

Não houve diferença significativa em relação ao sexo e ao escore $\mathrm{z}$ peso/idade nas duas faixas etárias nas quais os alunos foram classificados. $\mathrm{O}$ escore $\mathrm{z}$ altura/idade foi significativamente menor nas crianças da faixa etária maior (Tabela 2).

Tabela 2 - Comparação das características gerais dos alunos nas duas faixas etárias

\begin{tabular}{lccc}
\hline Variáveis & $\begin{array}{c}\mathbf{8 - 1 2} \text { anos } \\
\mathbf{n}(\boldsymbol{\%})\end{array}$ & $\begin{array}{c}\mathbf{1 3 - 1 8} \text { anos } \\
\mathbf{n}(\boldsymbol{\%})\end{array}$ & $\mathbf{p}$ \\
\hline $\begin{array}{l}\text { Sexo } \\
\quad \text { Masculino }\end{array}$ & & \\
Feminino & $57(39,9)$ & $34(41,5)$ & 0,924 \\
& $86(60,1)$ & $48(58,5)$ & \\
Índice (escore $\mathbf{z} \pm$ desvio-padrão) & & \\
$\begin{array}{l}\text { Peso/idade } \\
\text { Altura/idade }\end{array}$ & $0,15 \pm 1,11$ & $-0,06 \pm 1,03$ & 0,183 \\
& $-0,04 \pm 0,98$ & $-0,39 \pm 0,93$ & 0,009 \\
\hline
\end{tabular}

O teste do hidrogênio expirado foi positivo em 19 dos 225 pacientes estudados, o que demonstra uma prevalência de má absorção de doses fisiológicas de lactose de 8,4\% (IC 95\%: 5,2-12,9).

As médias dos valores de hidrogênio expirado encontrados no jejum e aos 60, 120 e 180 minutos após a ingestão de lactose nos 19 alunos com testes positivos estão demonstradas na Figura 1. 


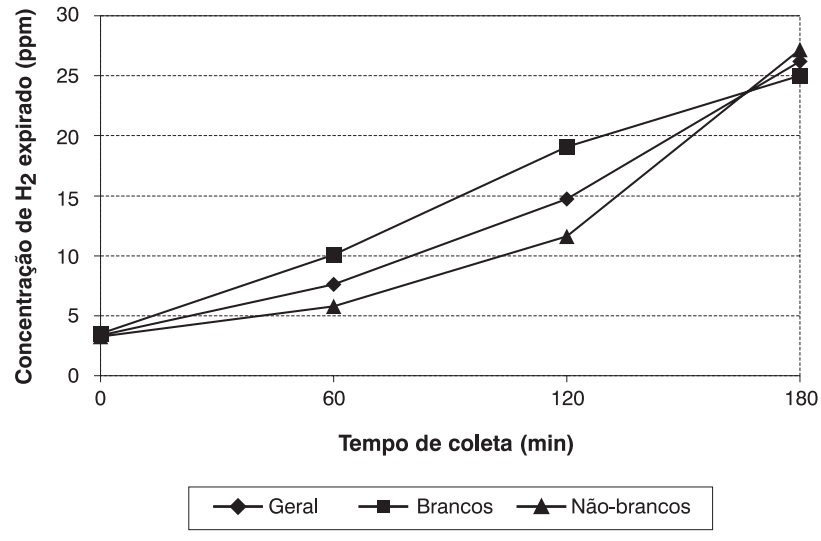

Figura 1 - Valores médios da concentração de hidrogênio expirado nos alunos com testes positivos

O maior aumento dos níveis de hidrogênio em relação ao valor basal nos exames positivos variou de 21 a 44, com média de $26,2 \pm 5,7$. Esse pico no delta $\mathrm{H}_{2}$ ocorreu aos 120 minutos em 3 dos 19 indivíduos (16\%), e aos 180 minutos nos 16 restantes (84\%). Já o primeiro aparecimento de um delta $\mathrm{H}_{2}$ acima de 20 se verificou em 1 indivíduo aos 60 minutos (5\%), em 3 aos 120 minutos (16\%), e em 15 aos 180 minutos $(79 \%)$.

A diferença nas taxas de prevalência de má absorção de lactose nos alunos de cor branca e não-branca $(5,2 \% \mathrm{x}$ $15,5 \%)$ foi estatisticamente significativa $(\mathrm{RP}=3,0 ; \mathrm{IC} 95 \%=$ $1,3-7,1 ; \mathrm{p}=0,02)$ (Tabela 3$)$.

Tabela 3 - Prevalência de má absorção de lactose de acordo com a cor

\begin{tabular}{lllllll}
\hline Cor & $\mathbf{n}$ & $\mathbf{f}$ & $(\%)$ & RP & IC95\% & $\mathbf{p}$ \\
\hline Branca & 154 & 8 & 5,2 & & & \\
Não-branca & 71 & 11 & 15,5 & 3,0 & $1,3-7,1$ & 0,020 \\
\hline
\end{tabular}

$\mathrm{n}$ : número de indivíduos estudados

f: número absoluto de indivíduos com má absorção

RP: razão de prevalência

IC: intervalo de confiança

Em relação à prevalência de má absorção de lactose de acordo com a faixa etária, não foi observada diferença estatisticamente significativa. Houve má absorção em 4,9\% dos alunos de 13 a 18 anos, e em 10,5\% dos alunos de 8 a 12 anos (RP= 0,47; IC95\%=0,16-1,35; p= 0,227) (Tabela 4). Após estratificação pela cor, encontramos, entre os alunos de cor branca, $6,9 \%$ de má absorção na faixa etária dos 8 aos 12 anos, e 1,9\% na compreendida entre 13 e 18 anos. Nos alunos de cor não-branca, as prevalências encontradas foram de $19 \%$ dos 8 aos 12 anos, e de $10,3 \%$ dos 13 aos 18 anos $(\mathrm{p}=0,506)$.

Tabela 4 - Prevalência de má absorção de lactose de acordo com a faixa etária

\begin{tabular}{lllllll}
\hline Faixa etária & $\mathbf{n}$ & $\mathbf{f}$ & $(\boldsymbol{\%})$ & RP & IC95\% & $\mathbf{p}$ \\
\hline 8-12 anos & 143 & 15 & 10,5 & & & \\
$\mathbf{1 3 - 1 8}$ anos & 82 & 4 & 4,9 & 0,47 & $0,16-1,35$ & 0,227
\end{tabular}

$\mathrm{n}$ : número de indivíduos estudados

f: número absoluto de indivíduos com má absorção

RP: Razão de prevalência

IC: Intervalo de confiança

\section{Discussão}

Nosso estudo avaliou a prevalência de má absorção de lactose através do teste do hidrogênio expirado, com a utilização de $250 \mathrm{ml}$ de leite de vaca integral. O teste teve duração de 3 horas, com coletas aos 60, 120 e 180 minutos após a ingestão do leite. Com a utilização de lactose na forma de leite ao invés de solução aquosa, as coletas devem ser prolongadas por 3 a 5 horas, devido ao retardo no esvaziamento gástrico e na excreção de $\mathrm{H}_{2}{ }^{13,14}$ As coletas de hidrogênio expirado em nosso estudo foram realizadas com intervalos de 60 minutos. Conforme demonstrado por Solomons et al. ${ }^{15}$, esse intervalo não diminui a sensibilidade do exame, mesmo com o uso de doses baixas $(12,5 \mathrm{~g})$ de lactose.

Consideramos como critério de positividade o aumento na concentração de hidrogênio igual ou superior a 20 ppm de hidrogênio em relação ao nível basal. Na literatura pediátrica, esse é o critério mais adotado devido a sua maior especificidade ${ }^{11,16-19}$ Alguns autores, no entanto, utilizam como ponto de corte um aumento do hidrogênio acima de $10 \mathrm{ppm}^{20,21}$

A prevalência de má absorção de doses fisiológicas de lactose em crianças e adolescentes de 8 a 18 anos, encontrada neste estudo, foi de $8,4 \%$. Considerando as duas faixas etárias em que a amostra foi dividida, a prevalência foi de $10,5 \%$ dos 8 aos 12 anos, e de $4,9 \%$ dos 13 aos 18 anos (Tabela 4).

Existem poucas publicações brasileiras sobre má absorção de lactose em crianças. Além disso, a lactose comumente é administrada em doses maiores e na forma de solução aquosa, o que dificulta a comparação de nossos dados, já que o emprego do leite otimiza sua absorção.

Reis et al. ${ }^{19}$ realizaram um estudo que avaliou a prevalência de má absorção em alunos de uma escola situada na periferia da cidade de Marília, São Paulo. Foram incluídos 
83 escolares, cuja idade variou de 7 a 15 anos. $\mathrm{O}$ método diagnóstico foi o teste do hidrogênio expirado após uma dose de $18 \mathrm{~g}$ de lactose em solução aquosa a 10\%. Com um ponto de corte de delta $\mathrm{H}_{2}$ acima de $20 \mathrm{ppm}, 19(22,9 \%)$ alunos foram classificados como maus absorvedores de lactose. Apesar do uso de doses semelhantes, a prevalência encontrada por esses autores é superior à obtida em nosso estudo. No entanto, os veículos utilizados para a administração da lactose foram diferentes.

Outra pesquisa com crianças brasileiras foi realizada em Minas Gerais, no município de Rio Acima, por Figueiredo $^{22}$, que avaliou 435 crianças de 7 a 15 anos de idade através do teste do hidrogênio expirado, após ingestão de $25 \mathrm{~g}$ de lactose em solução aquosa a $10 \%$. A prevalência de má absorção foi de $49,2 \%$, e de intolerância, de $24,3 \%$. Os resultados desse estudo também não são comparáveis aos nossos devido às diferenças na dose de lactose e no veículo empregados.

Alves et al. ${ }^{23}$ estudaram 251 crianças de duas aldeias índias no Mato Grosso do Sul e encontraram 34,5\% de má absorção de lactose nas maiores de quatro anos. Nas 54 crianças com menos de quatro anos, nenhum caso foi diagnosticado. O método utilizado para o dianóstico foi o teste do hidrogênio expirado, e foram administradas $18 \mathrm{~g}$ de lactose em solução aquosa a $10 \%$.

Nossos dados são semelhantes aos de um estudo realizado por Pitzalis et al. ${ }^{24} \mathrm{em}$ Roma, na Itália. Esses autores encontraram, em 70 crianças de 8 a 15 anos, uma prevalência de $5,7 \%$ de má absorção de lactose, com o uso de $250 \mathrm{ml}$ de leite.

Taxas menores de má absorção, com a utilização de doses semelhantes de leite, foram verificadas na Grécia por Ladas et al. ${ }^{16}$, em 150 crianças dos 5 aos 12 anos. Todas elas foram submetidas a dois testes: o primeiro com lactose na dose de $2 \mathrm{~g} / \mathrm{kg}$, e o segundo, 4 semanas após, com $240 \mathrm{ml}$ de leite. As taxas de prevalência de má absorção foram de $46 \%$ com o primeiro e de $2 \%$ com $240 \mathrm{ml}$ de leite. Extrapolando esses resultados para nossa população, podemos pressupor taxas de má absorção muito mais altas, caso houvéssemos empregado doses maiores de lactose.

Em relação à cor da pele dos alunos, nosso estudo demonstrou taxas de má absorção três vezes maiores nos alunos de cor não-branca, quando comparados aos de cor branca (Tabela 3). Em São Paulo, Troncon et al. ${ }^{25}$ também encontraram taxas mais elevadas de má absorção de lactose em pacientes de cor não-branca em relação aos de cor branca $(94,45 \%$ x $68,75 \%)$. Tais dados foram obtidos em pacientes adultos e com a utilização do teste de tolerância à lactose. Outro estudo efetuado em São Paulo por SevaPereira et al. ${ }^{8}$, em adultos, demonstrou taxas de $85 \%$ de má absorção em indivíduos negróides, e de 50\% em caucasóides. O estudo de Figueiredo ${ }^{22}$, realizado em Minas Gerais, com 435 crianças, também demonstrou taxas maiores de má absorção em crianças de cor preta $(60,3 \%)$ e parda $(50,7 \%)$ em relação às crianças de cor branca $(37,5 \%)$.
Em nosso estudo não foi demonstrada associação entre as taxas de má absorção de lactose e a faixa etária dos alunos. Encontramos 10,5\% de má absorção na faixa etária de 8 a 12 anos, e 4,9\% na compreendida entre 13 e 18 anos (Tabela 4). Embora a diferença não tenha alcançado significância estatística, houve tendência a uma menor proporção de casos na faixa etária mais elevada. Este achado paradoxal de menores taxas de má absorção com o aumento da idade também ocorreu em outros estudos, comparando faixas etárias semelhantes ${ }^{4,26}$. No estudo de Leis et al. ${ }^{6}$, a prevalência de má absorção de lactose encontrada na faixa etária de 6 a 13 anos $(36,4 \%)$ foi muito semelhante à encontrada dos 14 aos 18 anos (36,5\%). Esta tendência encontrada em nosso estudo poderia ser explicada caso houvesse uma maior proporção de crianças de cor nãobranca na faixa etária de 8 a 12 anos. Entretanto, mesmo com a estratificação pela cor, a tendência se manteve. Houve uma diferença significativa em um dos indicadores do estado nutricional, no caso o escore $\mathrm{z}$ altura/idade, entre as duas faixas etárias consideradas (Tabela 2). Uma maior prevalência de desnutrição severa nos alunos de 8 aos 12 anos poderia explicar a maior proporção de casos nessa faixa etária. No entanto, o escore $\mathrm{z}$ altura/idade foi menor nos alunos de 13 a 18 anos, faixa etária que apresentou taxas menores de má absorção de lactose. Além do efeito do acaso, que pode ter contribuído para estes resultados, já que a diferença não alcançou significância estatística $(\mathrm{p}=0,227)$, outro fator pode estar implicado. É possível que uma parcela dos indivíduos com intolerância à lactose e, portanto, má absorção, tenha se recusado a participar da pesquisa. A taxa de exclusão foi significativamente maior no grupo de 13 a 18 anos. Este viés de seleção pode ter excluído uma maior proporção de alunos com má absorção nesta faixa etária e, conseqüentemente, na amostra como um todo. Nesse caso, as taxas de má absorção por nós encontradas podem ter sido subestimadas.

Uma limitação do estudo foi o fato de não testarmos a capacidade de produção de hidrogênio pela flora bacteriana dos indivíduos incluídos. No entanto, há autores que demonstram uma proporção de até $9,2 \%$ de crianças colonizadas por flora incapaz de produzir hidrogênio 27 .

Nosso estudo analisou a prevalência de má absorção de lactose e sua associação com a cor da pele e a idade dos alunos. Na faixa etária estudada, a causa mais comum de má absorção de lactose é a hipolactasia primária do tipo adulto. Não podemos descartar, entretanto, que alguns casos diagnosticados nesta pesquisa também possam ser devidos à deficiência secundária de lactase. Contudo, a maioria das crianças e adolescentes estudados não apresentava desnutrição ou doenças crônicas, e os casos de diarréia foram excluídos.

A prevalência de má absorção de lactose encontrada com a utilização de $250 \mathrm{ml}$ de foi de $8,4 \%$. A comparação de nossos resultados foi dificultada pela escassez de estudos brasileiros semelhantes. Este estudo confirmou a associação entre má absorção de lactose e cor da pele. No entanto, 
não foi encontrada associação entre má absorção de lactose e faixa etária. O teste do hidrogênio expirado é um método simples, não invasivo, ideal para aplicação em estudos epidemiológicos.

\section{Referências bibliográficas}

1. Auricchio S, Troncone R. Genetically determined disaccharidase deficiencies. In: Walker WA, Durie P, Hamilton JR, WalkerSmith JA, Watkins JB, eds. Pediatric Gastrointestinal Disease. Ontario: BC Decker Inc; 2000.p.677-700.

2. Sahi T. The inheritance of selective adult-type lactose malabsorption. Scand J Gastroenterol 1974; 9 Suppl 30:1-73.

3. Sahi T, Launiala K. More evidence for the recessive inheritance of selective adult type lactose malabsorption. Gastroenterology 1977; 73:231-2.

4. Chang MH, Hsu HY, Chen C, Lee CH, Hsu JY. Lactose malabsorption and small intestinal lactase in normal chinese children. J Pediatr Gastroenterol Nutr 1987; 6:369-72.

5. Wittenberg DF, Moosa A. Lactose maldigestion: increased agerelated prevalence in institucionalized children. J Pediatr Gastroenterol Nutr 1990; 11:489-95.

6. Leis R, Tojo R, Pavón P, Douwes A. Prevalence of lactose malabsorption in Galicia. J Pediatr Gastroenterol Nutr 1997; 25:296-300.

7. Bayless TM, Rosensweig NS. A racial difference in incidence of lactase deficiency. JAMA 1966; 197:968-72.

8. Sevá-Pereira A, Magalhães AF, Pereira RA. Teste de sobrecarga com lactose no diagnóstico de malabsorção primária de lactose do adulto. Rev Bras Pat Clin 1982; 18:1-6.

9. Sevá-Pereira A, Silva RCMA, Pereira-Filho RA. Medida do $\mathrm{H}_{2}$ expirado no diagnóstico da má absorção de lactose. Arq Gastroenterol 1999; 36:18-26.

10. Perman JA, Montes RG. Breath analysis. In: Walker A, Durie PR, Hamilton RJ, Walker-Smith JA, Watkins JB, eds. Pediatric Gastrointestinal Disease. St Louis: Mosby Year Book; 1996. p.1635-45.

11. Rosado JI, Gonzales C, Valencia ME, Lopez P, Palma M, López $\mathrm{B}$, et al. Lactose maldigestion and milk intolerance: a study in rural and urban Mexico using physiological doses of milk. J Nutr 1994; 124:1052-9.

12. Victora CG, Barros FC, Vaughan JP. Crescimento e desnutrição. In: Epidemiologia da desigualdade. São Paulo: Hucitec; 1989. p.94-116.

13. Barillas-Mury C, Solomons NW. Test-retest reproducibility of hydrogen breath test for lactose maldigestion in preschool children. J Pediatr Gastroenterol Nutr 1987; 6:281-5.

14. Rosado JL, Solomons NW. Sensitivity and specificity of the hydrogen breath analysis test for detecting malabsorption of physiological doses of lactose. Clin Chem 1983; 29:545-8.

15. Solomons NW, García-Ibañez, Viteri FE. Hydrogen breath test of lactose absorption in adults: the application of physiological doses and whole cow's milk sources. Am J Clin Nutr 1980; 33:545-54.
16. Ladas SD, Katsiyiannaki-Latoufi E, Raptis SA. Lactose maldigestion and milk intolerance in healthy Greek schoolchildren. Am J Clin Nutr 1991; 53:676-80.

17. Solomons NW, Barillas C. The cut-off criterion for a positive hydrogen breath test in children: a reappraisal. J Pediatr Gastroenterol Nutr 1986: 5:920-5.

18. Veligati N, Treem W, Sullivan B, Burke G, Hyams JS. 10 ppm versus $20 \mathrm{ppm}$ : a reappraisal of diagnostic criteria for breath hydrogen testing in children. Am J Gastroenterol 1994; 89:758-61.

19. Reis JC, Morais MB, Fagundes-Neto U. Teste do hidrogênio no ar expirado na avaliação de absorção de lactose e sobrecrescimento bacteriano no intestino delgado de escolares. Arq Gastroenterol 1999; 36:169-76.

20. Abramowitz A, Granot E, Tamir I, Deckelbaum RJ. Two hour lactose breath hydrogen test. J Pediatr Gastroenterol Nutr 1986; 5:130-3.

21. Bustamante C, Mayans JAR, Socorro OM, Solano F, Garnica R, Garcia CIO, et al. Absorcion intestinal deficiente de lactosa en una poblacion de niños mexicanos sanos por medio de la prueba de iones hidrogeno en aire espirado. Acta Gastroent Latinoam 1996; 26:247-9.

22. Figueiredo RCP. Absorção e tolerância à lactose na população de escolares do município de Rio Acima-MG [tese]. Belo Horizonte: Universidade Federal de Minas Gerais; 2000.p.200.

23. Alves GM, Morais MB, Fagundes-Neto U. Absorção e tolerância à lactose por crianças índias terenas do Mato Grosso do Sul. Anais do X Congresso Brasileiro de Gastroenterologia PediátricaBrasília, DF, Brasil; 2001. p.40.

24. Pitzalis G, Fancellu MG, Deganello F, Galastri E, Bonamico M, Imperato C. Prevalence of lactose malabsorption in Roman school children. Minerva Pediatr 1993; 45:389-95.

25. Troncon LEA, Collares EF, Oliveira RB, Padovan W, Meneghelli UG. Mal-absorção de lactose em pacientes adultos do Hospital das Clínicas de Ribeirão Preto. Arq Gastroenterol 1981; 18:106-12.

26. Tadesse K, Leung DTY, Yuen RCF. The status of lactose absorption in Hong Kong chinese children. Acta Paediatr 1992; 81:598-600.

27. Douwes AC, Schaap C, Moorsel JM. Hydrogen breath test in schoolchildren. Arch Dis Child 1985; 60:333-7.

Endereço para correspondência:

Dra. Fernanda M. Pretto

Rua Dr. Rômulo Carbone, 412/803

CEP 95040-230 - Caxias do Sul, RS

Fone: (54) 99747898 - Fax: (54) 2221566

E-mail:fmpretto@terra.com.br 